\title{
Construção de um Sensor de Condutividade Elétrica do Solo: uma Proposta Multidisciplinar
}

\author{
Construction of a soil electrical conductivity sensor: a multidisciplinary proposal
}

Fernando Siqueira Scherer ${ }^{1}$, Silvana Maldaner ${ }^{2}$, Matheus Vinícius dos Santos Mello ${ }^{3}$, Priscila Bernardi Miranda ${ }^{4}$, Andrei da Cunha Lima ${ }^{5}$ e Adriano Rodrigues Luz ${ }^{6}$

1,2,3,4,5,6,Universidade Federal De Santa Maria, Cachoeira Do Sul, Brasil

Nandoscherer_@hotmail.com, silvana.maldaner@ufsm.br; bernardelipriscila@gmail.com cunhalimaandrei1@gmail.com; adrianorodrigues329@gmail.com

\section{Resumo}

A condutividade elétrica do solo é uma variável importante na agricultura. O seu conhecimento é imprescindível na fertirrigação e pode auxiliar no manejo do solo. Assim, a proposta deste trabalho é o desenvolvimento de um sensor para determinar a condutividade elétrica do solo a baixo custo. Para determinar a condutividade elétrica emprega-se a segunda lei de Ohm $(R=$ $\rho(l / A))$. A equação desta lei relaciona a resistência elétrica de um condutor, com o comprimento do fio,com a área de secção reta do fio e a resistividade (ou resistência especifica do material). Fisicamente, a condutividade elétrica ( $\sigma$ ) é o inverso da resistividade ( $\rho$ ). Desta forma, com um microcontrolador, um Arduino, um resistor de controle e placas condutoras para os eletrodos constroem-se um circuito simples para medição da condutividade elétrica. A tensão de entrada é $5 \mathrm{~V}$, a resistência do resistor de controle é $500 \mathrm{kohms}$, com estas informações calcula-se a corrente no circuito e determina-se a queda de tensão no circuito e consequentemente a condutividade elétrica do solo.

Palavras-chave: Condutividade; Sensor; Fertirrigação

\section{Abstract}

The electrical conductivity of the soil is an important variable in agriculture. Their knowledge is essential in fertirrigation and can assist in soil management1. Thus, the proposal of this work is the development of a sensor to determine the electrical conductivity of the soil at low cost. To determine the electrical conductivity, the second law of Ohm $(R=\rho(l / A))$ is used. The equation of this law relates the electrical resistance of a conductor, with the length of the wire, area of straight section of the wire and the resistivity (or specific resistance of the material). Physically, the electrical conductivity $(\sigma)$ is the inverse of the resistivity $(\rho)$. Thus, with a microcontroller, an Arduino, a control resistor and conductive plate electrodes, a simple circuit is created to measure the electrical conductivity. The input voltage is $5 v$, the resistance of the control resistor is 500kohms, with this information the current in the circuit is calculated and the voltage drop in the circuit and consequently the electrical conductivity of the ground is determined. 


\section{Introdução}

O conhecimento das características do solo é de fundamental importância em diversas áreas do conhecimento. Na agricultura diversos estudos apontam que tais características podem estar correlacionadas com as medidas elétricas do solo (Celinski , 2008), como a resistência, resistividade, condutividade e capacitância elétrica. Os estudos de Molin et al. (2003), Castro (2004), Pincelli (2004), apontam que o conhecimento da condutividade elétrica do solo é uma propriedade diretamente relacionada à produtividade e consequentemente à racionalização do uso de insumos.

Assim, diante da relevância da aplicabilidade deste sensor e considerando o seu alto custo quando comercializado, neste estudo propõe-se a elaboração de um circuito simples que permita a medida física da condutividade elétrica.

\section{Metodologia}

Para a construção do sensor de condutividade elétrica foi empregado dois eletrodos retangulares de $1 \mathrm{~cm}$ de largura, um resistor de controle 500 kohms e um microcontrolador para a leitura das medidas. A escolha do resistor de controle foi baseada no trabalho de Menegatti (1999). Este autor, em seu trabalho experimental com solo, conclui que para medir condutividade é necessário um resistor de aproximadamente $500 \mathrm{kohms}$ para eletrodo separados por $14 \mathrm{~cm}$. Adicionalmente, são utilizadas as leis de Ohm e a definição física que relaciona a condutividade elétrica à resistividade elétrica, conforme equação abaixo:

$$
\alpha=\frac{1}{\rho}
$$

Onde $\sigma$ representa a condutividade elétrica $\left(\frac{S}{m}\right)$ e $\rho$ a resistividade elétrica $(\Omega m)$.

\section{Resultados}

O principal resultado deste estudo foi a construção e análise de um circuito para medição da condutividade elétrica do solo a baixo custo. O circuito é apresentado na Figura 1.

Figura 1 - Circuito para a construção do sensor de condutividade elétrica

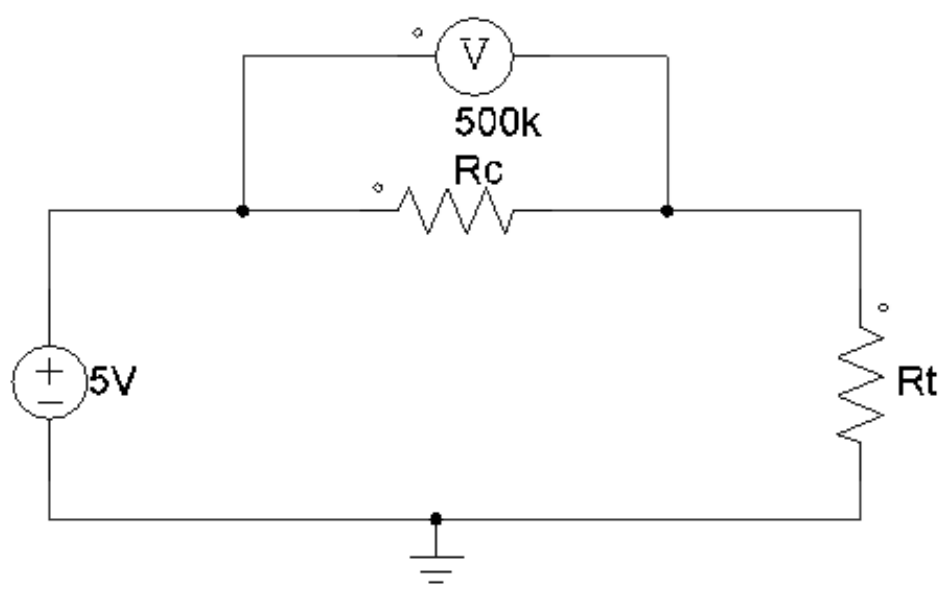


Utilizando o microcontrolador do arduino ATMEGA328P, uma tensão de $5 \mathrm{~V}$ é fornecida ao circuito. O resistor de controle de 500 kohms está em série com um resistor desconhecido, Rt. O resistor Rt representa a resistência do solo. Para efetuar as medições de resistência elétrica, os dois eletrodos são colocados na amostra de solo. Utilizando as portas analógicas do microcontrolador, mede-se o valor da queda de tensão no resistor de 500. Pela lei de Ohm, sabe-se que:

$i=\frac{V}{R}$

A equação acima mostra que a corrente elétrica pode ser calculada a partir da queda de potencial no resistor de controle. Como no circuito (Figura 1) os resistores estão em série, a corrente que percorre a malha é a calculada pela eq. (2). Além disso, a queda de tensão no circuito é determinada por:

$V_{t}=5 V-V_{500 \mathrm{~K} \Omega}$

Assim, com o valor da corrente e a queda de potencial na malha conforme eq. (3), pode-se determinar a resistência do solo:

$R_{t}=\frac{V_{t}}{i}$

Logo, a partir do valor da resistência elétrica calculada a partir da eq. (4) e da segunda lei de Ohm, juntamente com a eq. (1), determina-se a condutividade elétrica do solo.

\section{Considerações finais}

Com o presente trabalho conclui-se que é possível desenvolver um sensor de condutividade elétrica a baixo custo. Porém, nenhuma calibração deste sensor foi realizada e desta forma será necessário uma calibração experimental envolvendo diferentes tipos de solo para então ser utilizado em medições.

\section{Agradecimentos}

Os autores agradecem à Fundação de Amparo à Pesquisa do Estado do Rio Grande do Sul - FAPERGS (edital 01/2017 - ARD), ao Conselho de Desenvolvimento Científico e Tecnológico - CNPq, à Coordenadora do Polo UABCachoeira do Sul (Rosane Aparecida Brendler Keller), à Prefeitura Municipal de Cachoeira do Sul e ao Programa de Auxílio à pesquisa de recém-doutores "FIPE -Júnior/UFSM".

\section{Referências}

CASTRO CN. Definição de unidades de gerenciamento do solo por meio da sua condutividade elétrica e variáveis físicoquímicas, em Piracicaba/SP [dissertation]. Piracicaba: Universidade de São Paulo; 2004.131p.

MOLIN JP, GIMENEZ LM, PAULETTI V, SCHMIDHALTER U, HAMMER J. Mensuração da condutividade elétrica do solo por indução e sua correlação com fatores de produção. Engenharia Agrícola. 2005; 25(2): 420-426. 
NADAI HLL, NACHREINER ML, MENEGATTI LAA, MOLIN JP. Estudos preliminares de um sensor de condutividade elétrica do solo para aplicação em agricultura de precisão [Internet]. 2000. [cited 2018 mar 20]. Available from: http://www.ler.esalq.usp.br/download/gmap/iniciacao/EstudosPreliminaresdeumSensor2000.pdf>.

PINCELLI ALS. Desenvolvimento e ensaios de um sistema de mensuração de condutividade elétrica do solo, em Piracicaba/SP [dissertation]. Piracicaba: Escola Superior de Agricultura Luiz de Queiroz -Universidade de São Paulo; 2004. $87 \mathrm{p}$.

ROQUE, W. Desenvolvimento de um multi-sensor eletronico para medida da umidade, temperatura e condutividade elétrica do solo, em Campinas/SP[dissertation]. Campinas: Faculdade de Engenharia Elétrica e de Computação; 2008. 42p. 